ACS Infectious Disease - Supporting Information

\title{
Intrabacterial Metabolism Obscures the Successful Prediction of an InhA Inhibitor of Mycobacterium tuberculosis
}

Xin Wang, ${ }^{\dagger 1}$ Alexander L. Perryman, ${ }^{\dagger 1}$ Shao-Gang Li, ${ }^{1}$ Steve D. Paget, ${ }^{1}$ Thomas P. Stratton, ${ }^{1}$ Alex Lemenze,${ }^{2}$

Arthur J. Olson, ${ }^{3}$ Sean Ekins, ${ }^{4,5}$ Pradeep Kumar, ${ }^{2}$ and Joel S. Freundlich*1,2

$\dagger$ Contributed equally.

${ }^{1}$ Department of Pharmacology, Physiology, and Neuroscience, Rutgers University-New Jersey Medical School, Medical Sciences Building, 185 South Orange Avenue, Newark, NJ 07103, USA.

${ }^{2}$ Division of Infectious Disease, Department of Medicine, and the Ruy V. Lourenço Center for the Study of Emerging and Reemerging Pathogens, Rutgers University-New Jersey Medical School, Medical Sciences Building, 185 South Orange Avenue, Newark, NJ 07103, USA.

${ }^{3}$ Department of Integrative Structural and Computational Biology, The Scripps Research Institute, Room MB112/Mail Drop MB5, 10550 North Torrey Pines Road, La Jolla, CA 92037, USA.

${ }^{4}$ Collaborations in Chemistry, 5616 Hilltop Needmore Road, Fuquay-Varina, NC 27526, USA.

*Corresponding author : Joel S. Freundlich (reundjs@,rutgers.edu) 


\section{Table of Contents}

Figure S1. Dose-response curve for InhA inhibition by a) JSF-2149, b) BAS00131943, c), ASN06744915, d) ASN06744991, and e) JSF-2164.

Figure S2. Synthetic scheme for JSF-2149.

Figure S3. Synthetic scheme for JSF-2164 and its candidate metabolites.

Figure S4. InhA activity in the presence of Tween-20.

Figure S5. JSF-2149 exhibited detergent-dependent inhibition of purified InhA.

Figure S6. RIF in vitro activity versus the ss18b strain.

Figure S7. inh $A$ promoter mutation in 16x6 resulted in inhA over-expression.

Figure S8. LC-MS data supporting identification of JSF-3617 as intrabacterial metabolite M1.

Figure S9. LC-MS data supporting identification of JSF-3616 as intrabacterial metabolite M2.

Table S1. 370 compounds that passed the initial docking filters and were scored with two recently validated Bayesian dual-event models.

Table S2. Antitubercular activity of 5 lowest-scoring compounds amongst the 370 candidates passing docking filters.

Table S3. Profiling of select spontaneous JSF-2164-resistant mutants.

Table S4. MIC values for JSF-2164, INH, and pretomanid versus JSF-2164-resistant transposon mutants. 
Figure S1. Dose-response curve for InhA inhibition by A) JSF-2149, B) BAS00131943, C), ASN06744915,

D) ASN06744991, and E) JSF-2164. Error bars show standard errors of measurements with biological triplicates.
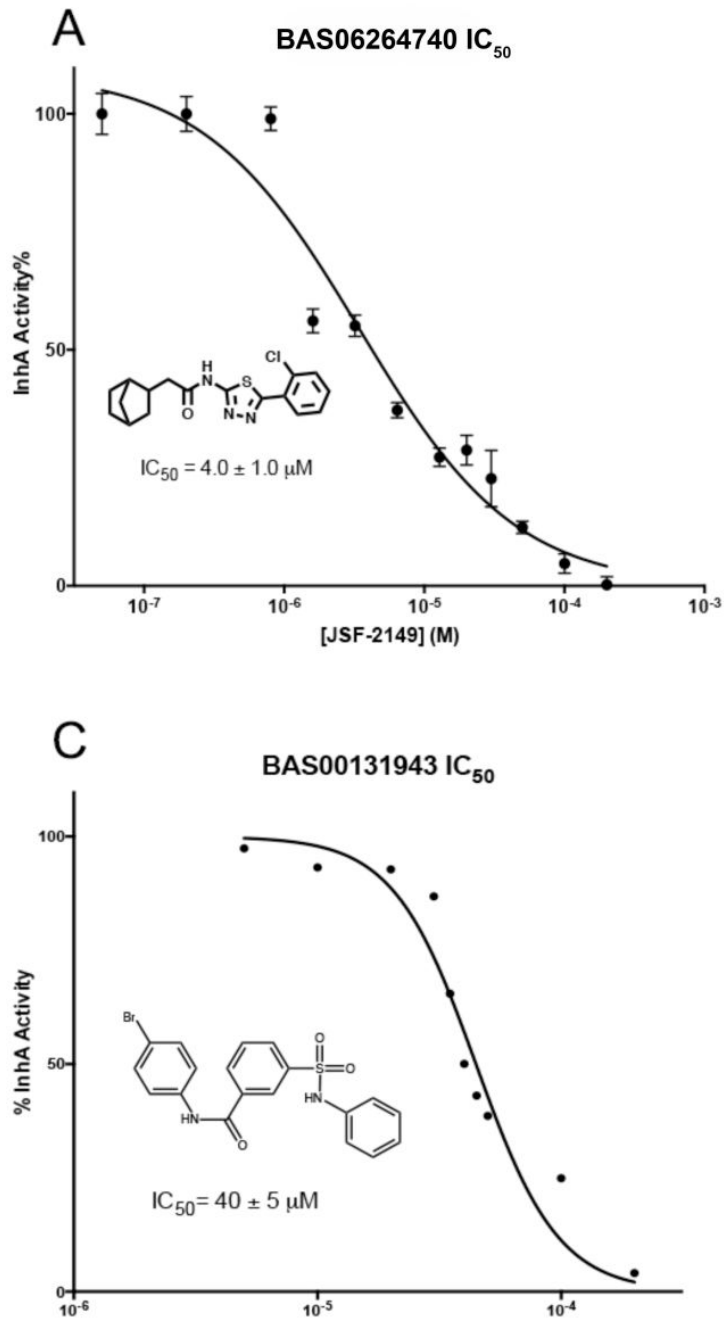

[BAS00131943] (M)

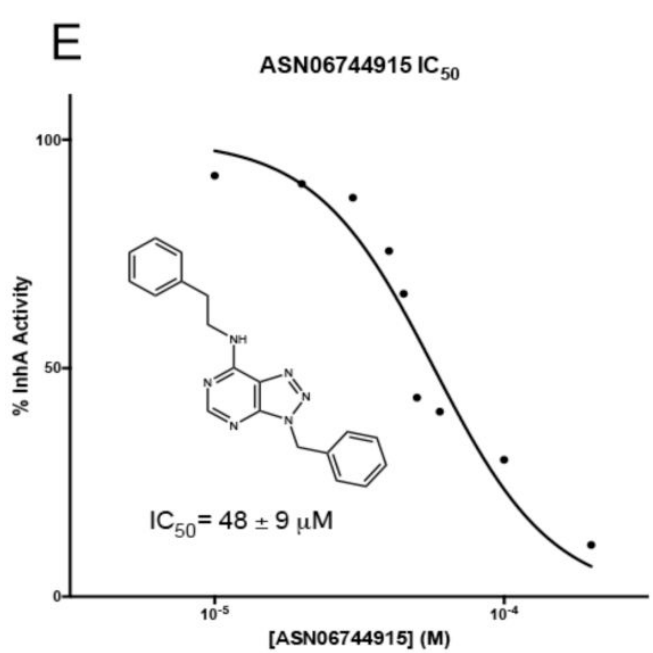

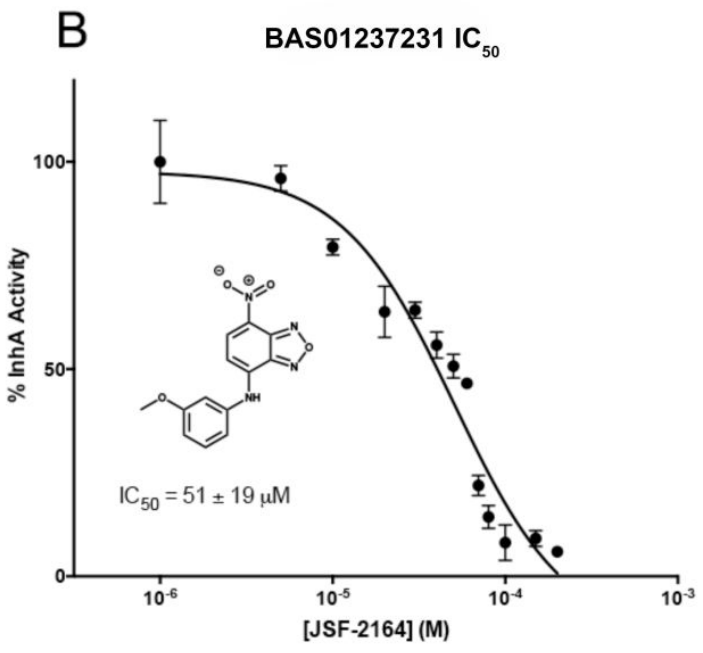

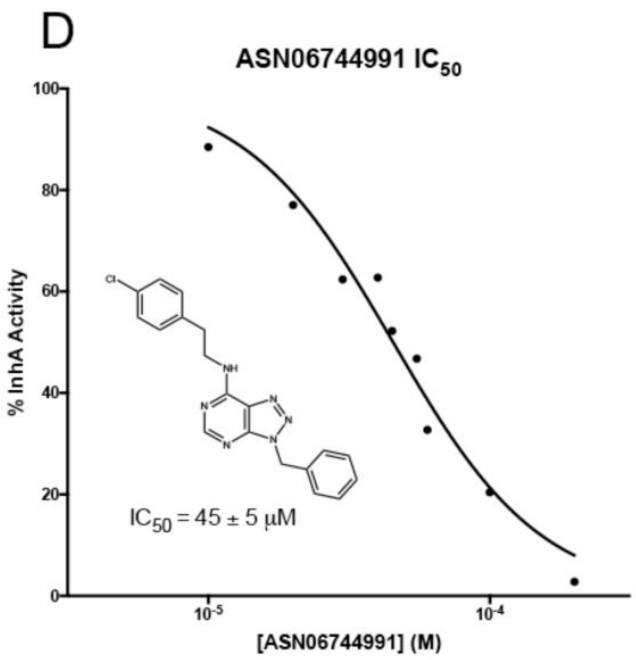

F

Triclosan $\mathrm{IC}_{50}$

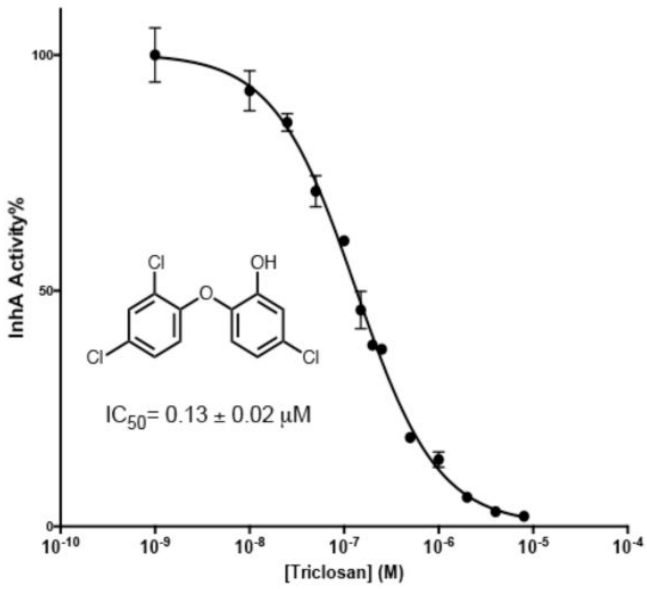

S3 
Figure S2. Synthetic scheme for JSF-2149.

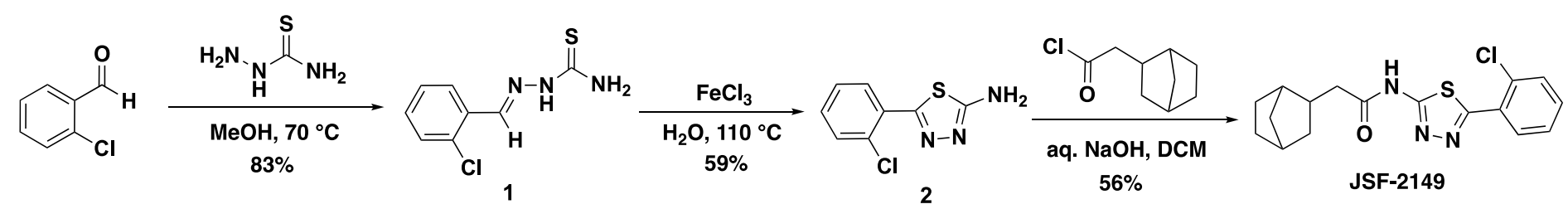


Figure S3. Synthetic scheme for JSF-2164 and its candidate metabolites.<smiles></smiles><smiles>COc1cccc(N)c1</smiles><smiles>COc1cccc(Nc2ccc([N+](=O)[O-])c3nonc23)c1</smiles><smiles>Nc1cccc2nonc12</smiles><smiles>COc1cccc(Br)c1</smiles><smiles>COc1cccc(Nc2cccc3nonc23)c1</smiles><smiles>COc1cccc(Nc2ccc([N+](=O)[O-])c3nonc23)c1</smiles><smiles>COc1cccc(Nc2ccc(N)c3nonc23)c1</smiles><smiles>O=[N+]([O-])c1c(Cl)ccc2nonc12</smiles><smiles>COc1cccc(N)c1</smiles><smiles>COc1cccc(Nc2ccc3nonc3c2[N+](=O)[O-])c1</smiles><smiles>[R]OC(F)(F)OC</smiles><smiles>COc1cccc(Nc2ccc3nonc3c2N)c1</smiles> 
Figure S4. InhA activity in the presence of Tween-20. InhA activity $(\mu \mathrm{M} / \mathrm{s})$ was measured at a range of concentrations of Tween-20 (0.001X $-1 \mathrm{X} \mathrm{CMC})$, where CMC $=60 \mathrm{mg} / \mathrm{L}$. InhA activity without Tween-20 was $0.10 \mu \mathrm{M} / \mathrm{s}$. InhA catalytic activity reached a plateau from $0.001 \mathrm{CMC}$ until approximately the detergent's CMC. Therefore, a range of Tween-20 concentrations from $0.01 \mathrm{CMC}$ to $0.5 \mathrm{CMC}$ may be used for testing compound aggregation. The error bars quantify the standard errors for each measurement made in biological triplicates.

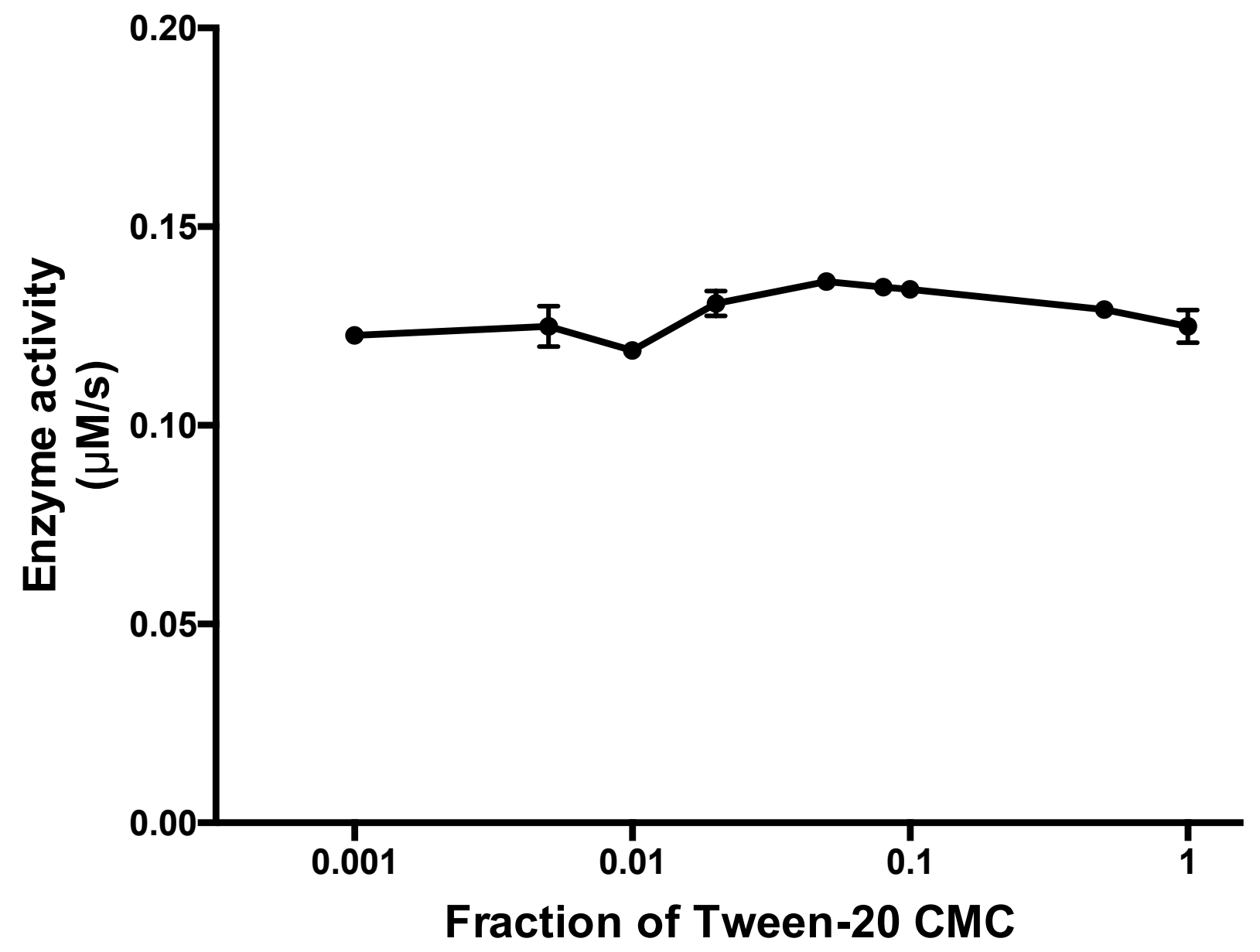


Figure S5. JSF-2149 exhibited detergent-dependent inhibition of purified InhA. InhA dose-response curves with or without $0.01 \mathrm{CMC}$ Tween-20 were generated and the $\mathrm{IC}_{50}$ was calculated as mean \pm standard error for (A) JSF-2149, (B) Triclosan as a negative control, and (C) benzyl benzoate as a positive control. The error bars showed standard errors of each measurement in biological triplicates.
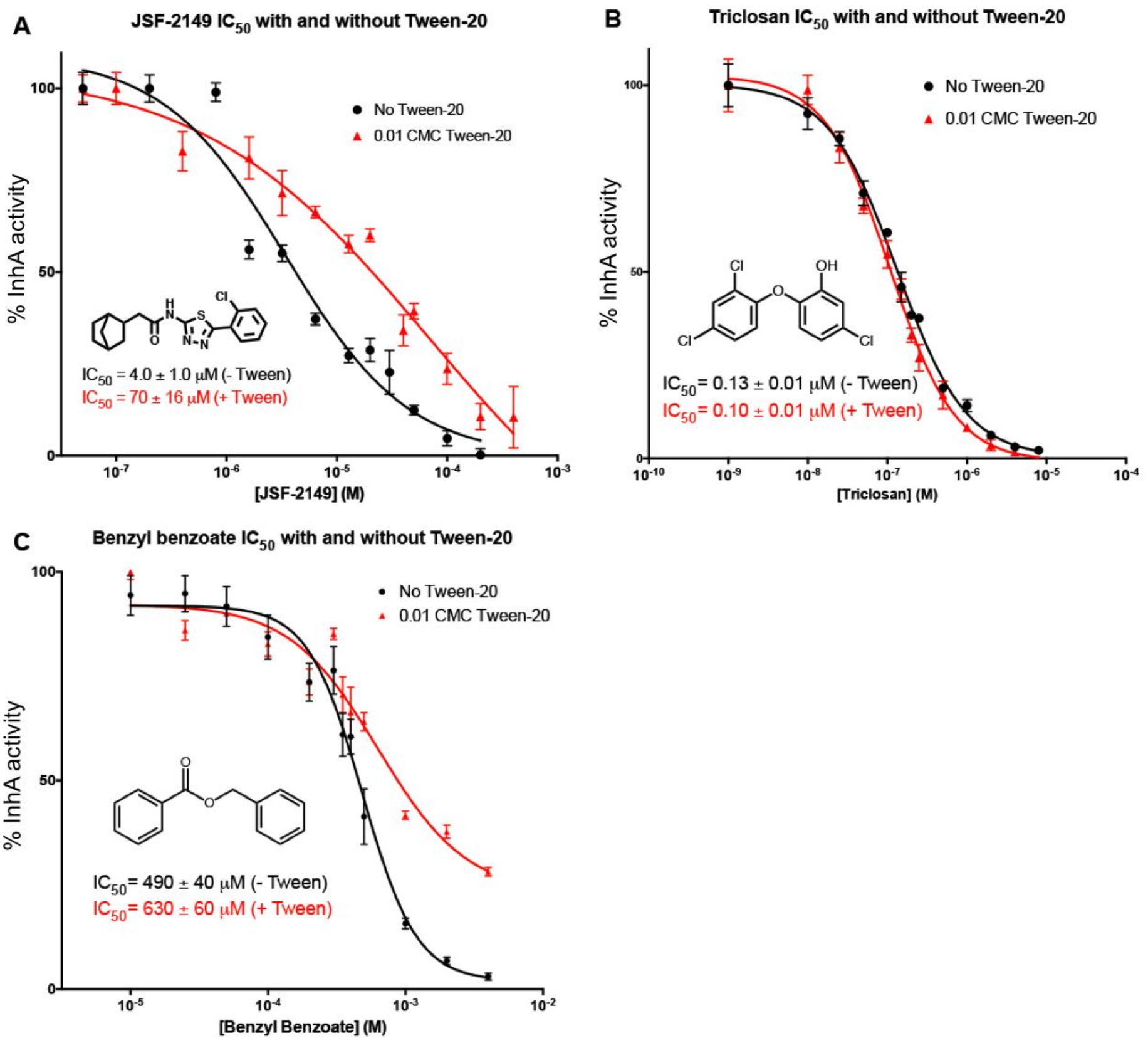
Figure S6. RIF in vitro activity versus the ss18b strain. Strain ss $18 \mathrm{~b}$ was starved without streptomycin supplementation in $7 \mathrm{H} 9+\mathrm{ADS}$ media for 2 weeks at $\mathrm{OD}_{595}=0.3$. RIF activity was then measured by REMA. The error bars showed standard errors of each measurement in biological triplicates.

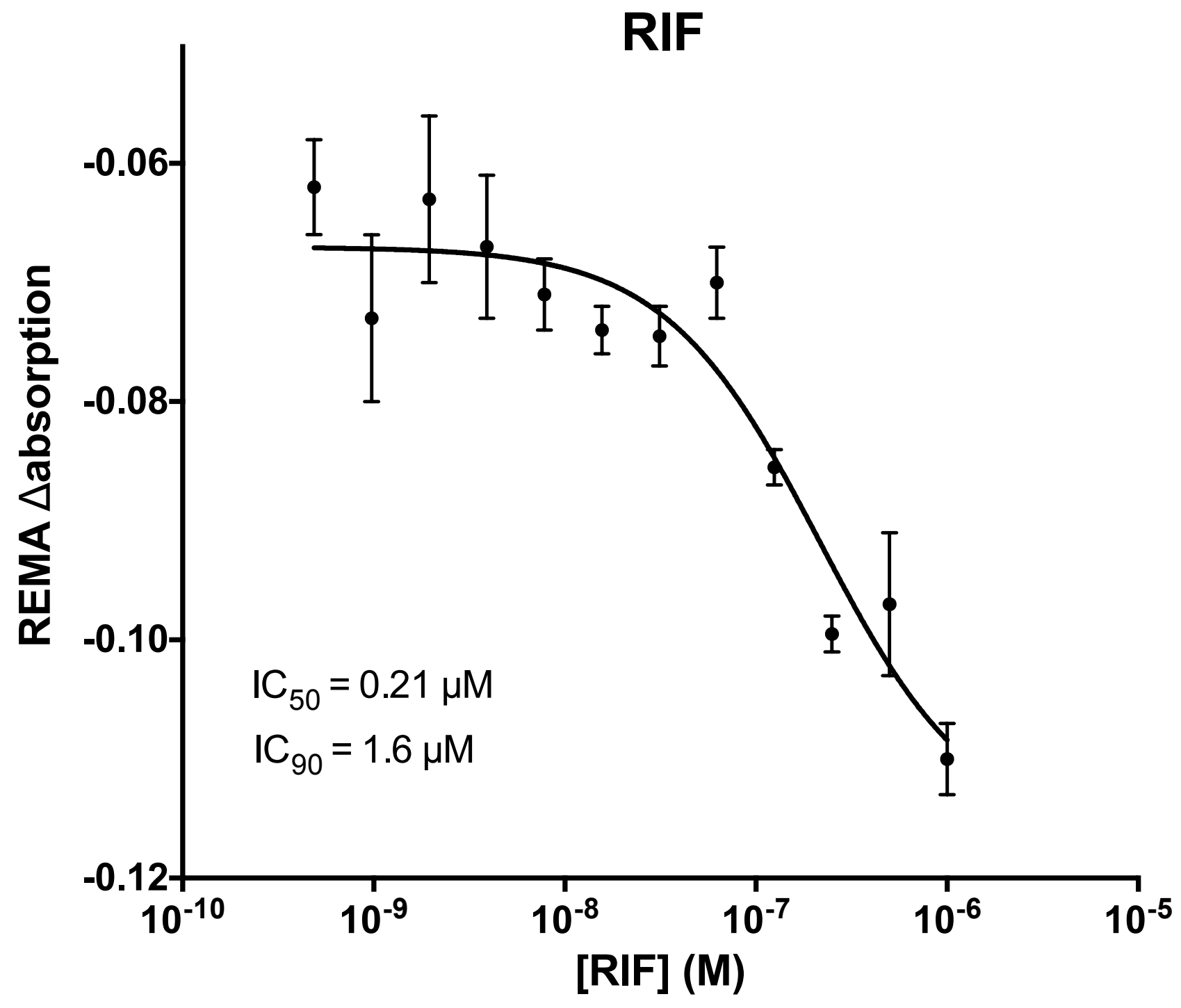


Figure S7. $\operatorname{inh} A$ promoter mutation in $16 x 6$ resulted in $i n h A$ over-expression. (A) Mutation $c(-15) t$ in strains 16x6 and $\mathrm{mc}^{2} 4914$ as determined by whole-genome and Sanger sequencing. (B) Expression levels of inh $A$ and $m a b A$ in mutant strains as compared to expression in $\mathrm{H} 37 \mathrm{Rv}$, as assayed via qPCR and quantified by expression of $16 \mathrm{~S}$ rRNA. The error bars showed standard errors of each measurement in biological triplicates. inhA and $m a b A$ expression in the mutant strains were compared to those in the wild type strain followed by statistical analysis with an unpaired Student's t-test. *** $p<0.001$

A
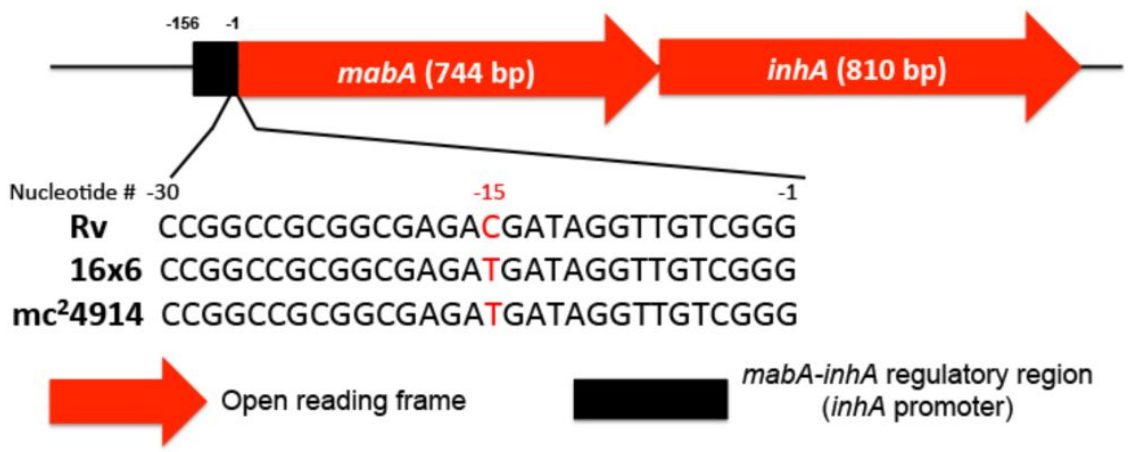

B

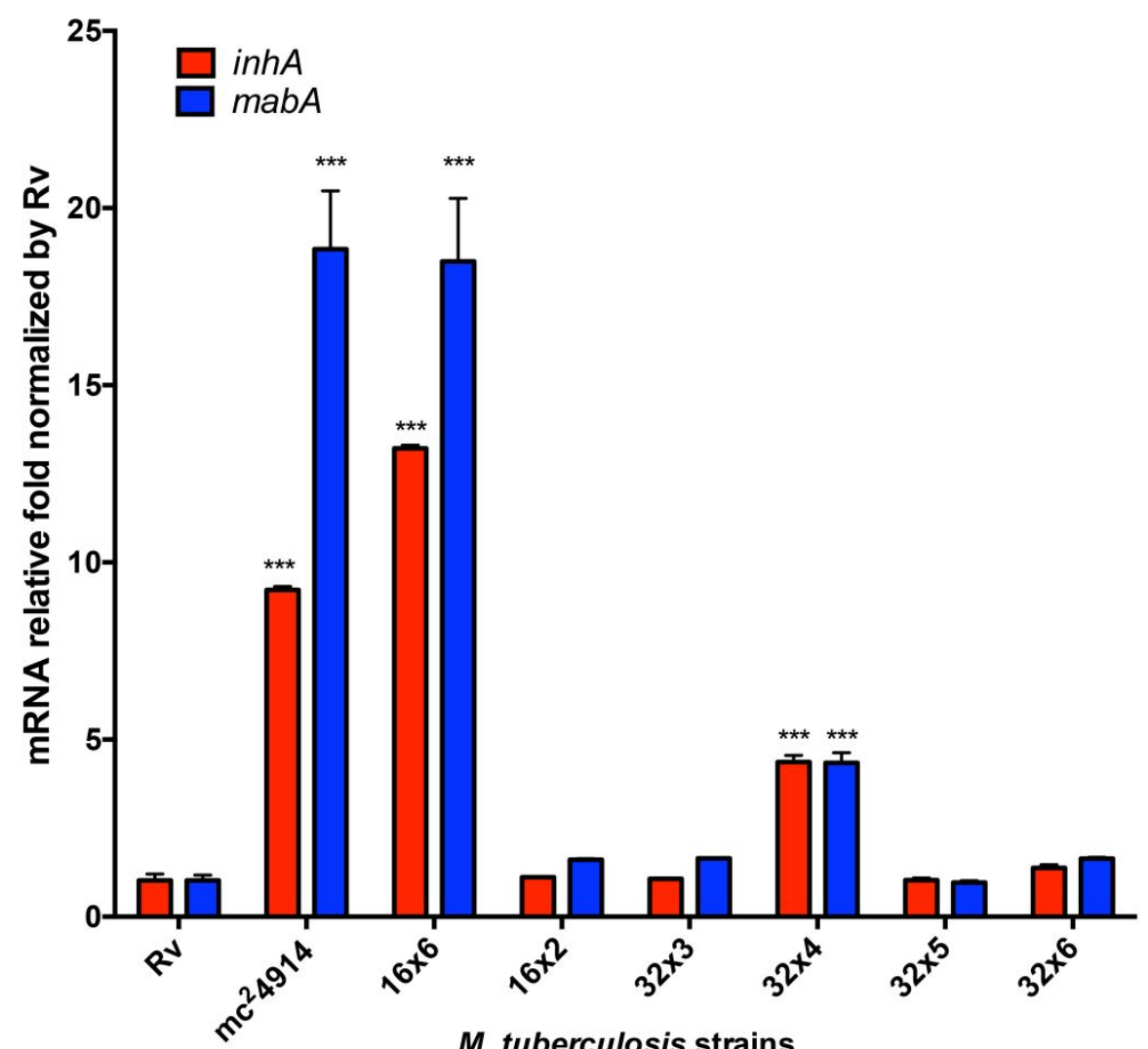


Figure S8. LC-MS data supporting identification of JSF-3617 as intrabacterial metabolite M1. Metabolite with $\mathrm{m} / \mathrm{z}$ of 257.1030 was purified from bacterial lysate followed by analysis via (A and B) high-resolution mass spectrometry and (C) LC-MS co-elution of isolated M1 with synthetic JSF-3617.

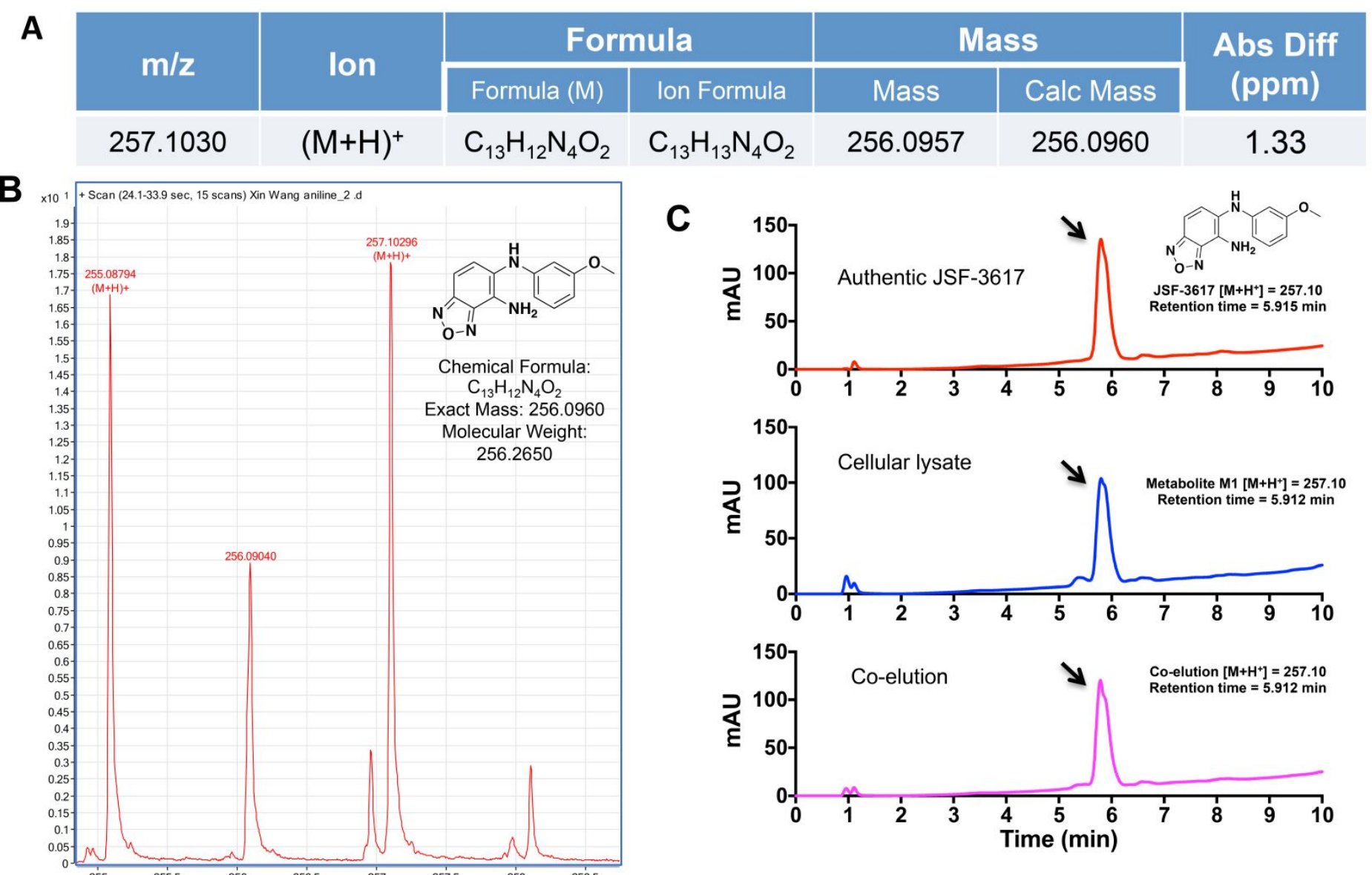


Figure S9. LC-MS data supporting identification of JSF-3616 as intrabacterial metabolite M2. Metabolite with $\mathrm{m} / \mathrm{z}$ of 257.1030 was purified from bacterial lysate followed by analysis via (A and B) high-resolution mass spectrometry and (C) LC-MS co-elution of isolated M2 with synthetic JSF-3616.

A

\begin{tabular}{|c|c|c|c|c|c|c|}
\hline \multirow{2}{*}{$\mathrm{m} / \mathrm{z}$} & \multirow{2}{*}{ Ion } & \multicolumn{2}{|c|}{ Formula } & \multicolumn{2}{c|}{ Mass } & \multicolumn{2}{c|}{$\begin{array}{c}\text { Abs Diff } \\
(\mathrm{ppm})\end{array}$} \\
\cline { 3 - 7 } & & Formula $(\mathrm{M})$ & lon Formula & Mass & Calc Mass & 1.58 \\
\hline 257.1030 & $(\mathrm{M}+\mathrm{H})^{+}$ & $\mathrm{C}_{13} \mathrm{H}_{12} \mathrm{~N}_{4} \mathrm{O}_{2}$ & $\mathrm{C}_{13} \mathrm{H}_{13} \mathrm{~N}_{4} \mathrm{O}_{2}$ & 256.0956 & 256.0960 & 1.58 \\
\hline
\end{tabular}

B

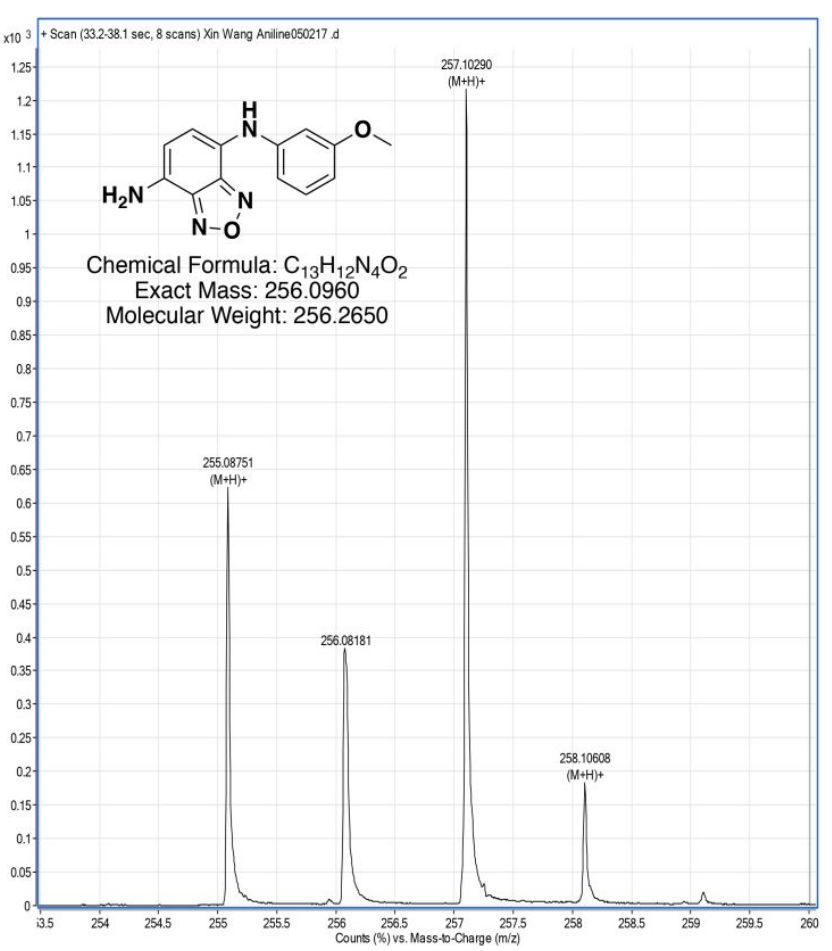

C
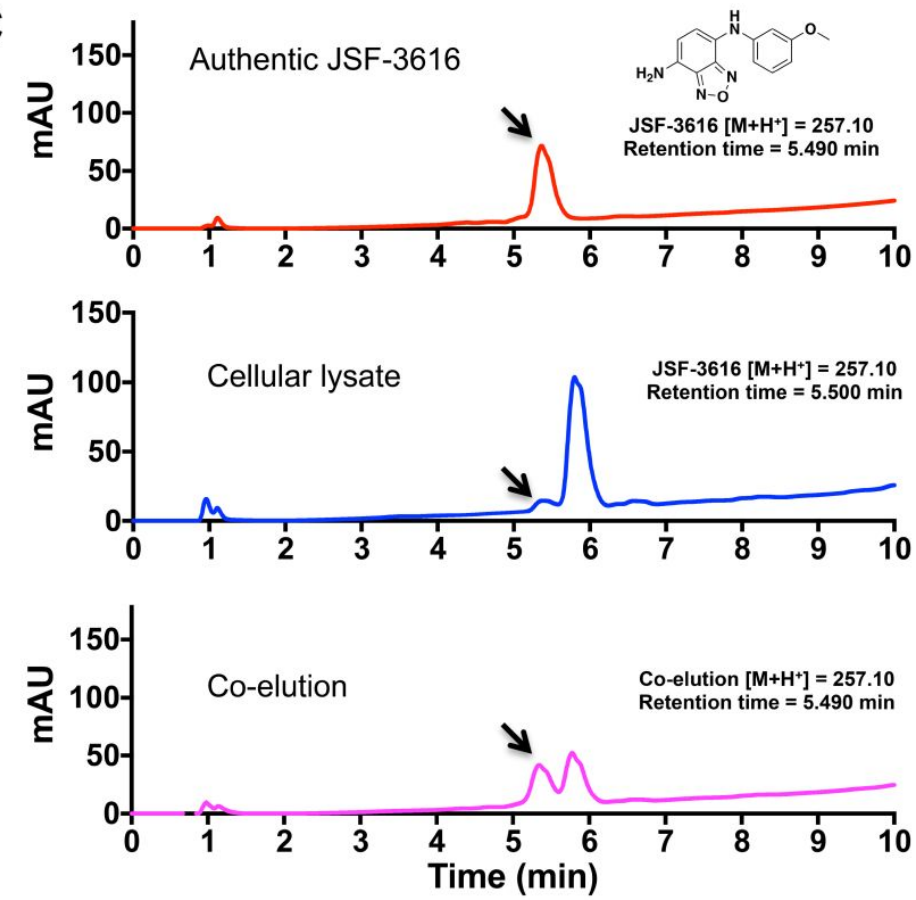
Table S1. 370 compounds that passed the initial docking filters and were scored with two recently validated Bayesian dual-event models.

Please view file : Table S1 DockingFiltered370_wCB2_nCombined.xlsx 
Table S2. Antitubercular activity of 5 lowest-scoring compounds amongst the 370 candidates passing docking filters.

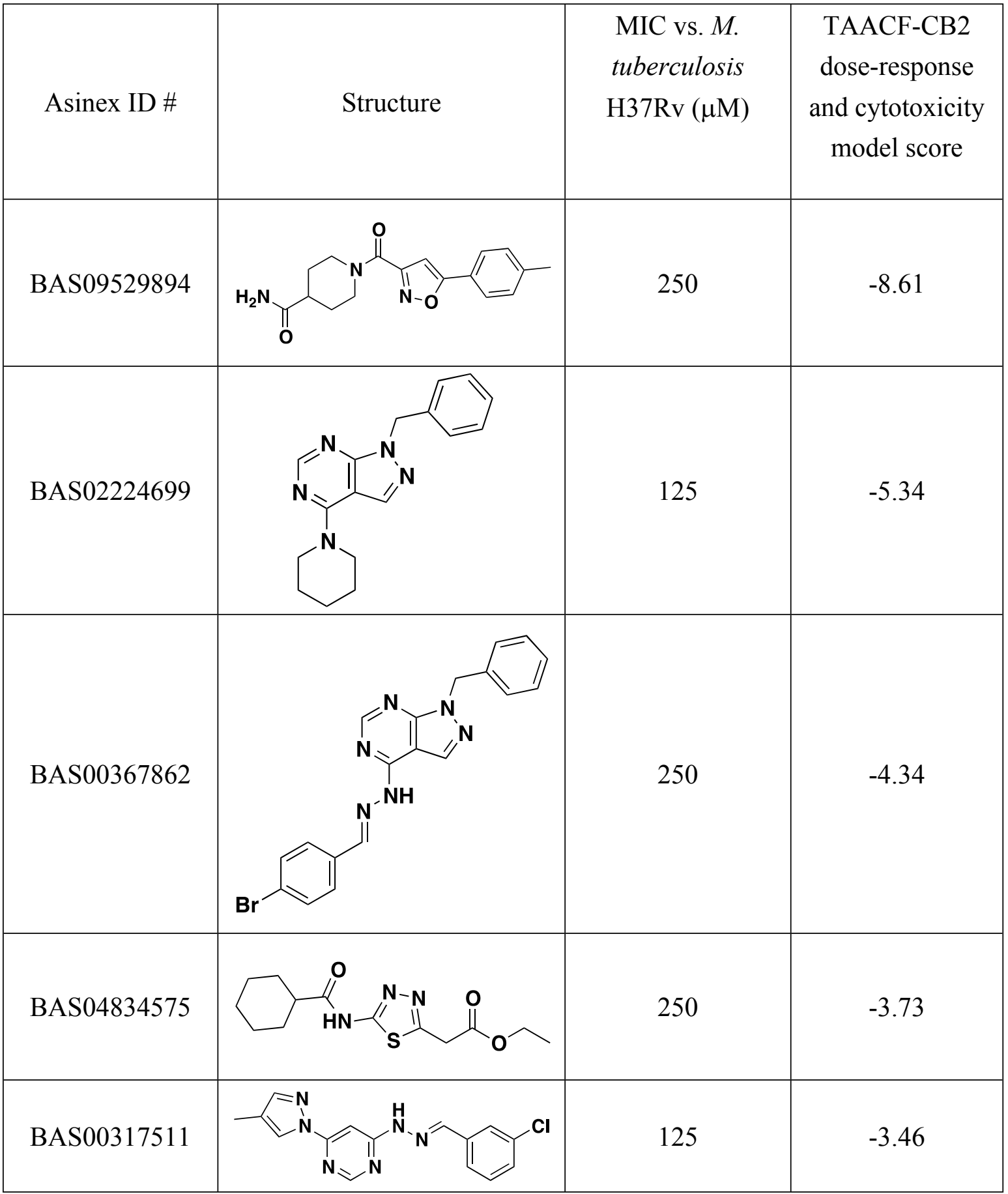


Table S3. Profiling of select spontaneous JSF-2164-resistant mutants.

\begin{tabular}{|l|l|l|l|l|}
\hline \multirow{2}{*}{ Strain } & Gene(s) mutated & \multicolumn{2}{l}{ MIC $(\boldsymbol{\mu M})$} \\
\cline { 3 - 5 } & (Amino acid change) & JSF-2164 & INH & Pretomanid \\
\hline H37Rv & $/$ & 8.0 & 0.16 & 0.078 \\
\hline $16 \times 2$ & $f b i C(\mathrm{~F} 567 \mathrm{~S})$ & 250 & 0.078 & $>40$ \\
\hline $16 \times 6$ & $f g d 1$ (frameshift at L138) & 250 & 1.25 & $>40$ \\
\hline $32 \times 4$ & inhA promoter $c(-15) t$ & & 1.25 & 0.16 \\
\hline $32 \times 5$ & tmk promoter $g(-66) \mathrm{t}$ & 31 & 0.64 & $>40$ \\
\hline
\end{tabular}


Table S4. MIC values for JSF-2164, INH, and pretomanid versus JSF-2164-resistant transposon mutants.

Please view file : Table S4 Transposon_MICs.xlsx 